\title{
Vagus nerve stimulation for depression: efficacy and safety in a European study
}

\author{
T. E. Schlaepfer ${ }^{1,2,3 *}$, C. Frick ${ }^{1,2}$, A. Zobel ${ }^{2}$, W. Maier ${ }^{2}$, I. Heuser ${ }^{4}$, M. Bajbouj ${ }^{4}$, V. O' Keane $^{5}$, \\ C. Corcoran $^{5}$, R. Adolfsson ${ }^{6}$, M. Trimble ${ }^{7}$, H. Rau ${ }^{8,9}$, H.-J. Hoff ${ }^{8}$, F. Padberg ${ }^{10}$, F. Müller-Siecheneder ${ }^{10}$, \\ K. Audenaert ${ }^{11}$, D. Van den Abbeele ${ }^{11}$, K. Matthews ${ }^{12}$, D. Christmas ${ }^{12}$, Z. Stanga ${ }^{1}$ and M. Hasdemir ${ }^{13}$ \\ ${ }^{1}$ Department of Psychiatry, University Hospital, Bern, Switzerland \\ ${ }^{2}$ Department of Psychiatry and Psychotherapy, University Hospital, Bonn, Germany \\ ${ }^{3}$ Departments of Psychiatry and Mental Health, The Johns Hopkins University, Baltimore, MD, USA \\ ${ }^{4}$ Psychiatrische Klinik der FU Berlin, Berlin, Germany \\ ${ }^{5}$ Beaumont Hospital, Department of Psychiatry, Dublin, Ireland \\ ${ }^{6}$ University Hospital Umea, Umea, Sweden \\ ${ }^{7}$ National Hospital for Neurology and Neurosurgery, Department of Neuropsychiatry and Neurology, London, UK \\ ${ }^{8}$ Evangelisches Krankenhaus Bielefeld, Bielefeld, Germany \\ ${ }^{9}$ Alcohol Rehabilitation Clinic, Wilhelmsdorf, Germany \\ ${ }^{10}$ Departments of Psychiatry and Psychotherapy, Ludwig-Maximilian-University, Munich, Germany \\ ${ }^{11}$ University Hospital Gent, Gent, Belgium \\ ${ }^{12}$ Ninewells Hospital and Medical School, Department of Psychiatry, Dundee, UK \\ ${ }^{13}$ Lindenhof Hospital, Department of Neurosurgery, Bern, Switzerland
}

Background. Vagus nerve stimulation (VNS) therapy is associated with a decrease in seizure frequency in partial-onset seizure patients. Initial trials suggest that it may be an effective treatment, with few side-effects, for intractable depression.

Method. An open, uncontrolled European multi-centre study (D03) of VNS therapy was conducted, in addition to stable pharmacotherapy, in 74 patients with treatment-resistant depression (TRD). Treatment remained unchanged for the first 3 months; in the subsequent 9 months, medications and VNS dosing parameters were altered as indicated clinically.

Results. The baseline 28-item Hamilton Depression Rating Scale (HAMD-28) score averaged 34. After 3 months of VNS, response rates $(\geqslant 50 \%$ reduction in baseline scores) reached $37 \%$ and remission rates (HAMD-28 score $<10$ ) $17 \%$. Response rates increased to $53 \%$ after 1 year of VNS, and remission rates reached $33 \%$. Response was defined as sustained if no relapse occurred during the first year of VNS after response onset; $44 \%$ of patients met these criteria. Median time to response was 9 months. Most frequent side-effects were voice alteration $(63 \%$ at 3 months of stimulation) and coughing (23\%).

Conclusions. VNS therapy was effective in reducing severity of depression; efficacy increased over time. Efficacy ratings were in the same range as those previously reported from a USA study using a similar protocol; at 12 months, reduction of symptom severity was significantly higher in the European sample. This might be explained by a small but significant difference in the baseline HAMD-28 score and the lower number of treatments in the current episode in the European study.

Received 12 April 2007; Revised 4 December 2007; Accepted 10 December 2007; First published online 4 January 2008

Key words: Brain stimulation, major depression, treatment resistance, vagus nerve stimulation.

\section{Introduction}

Antidepressant drugs, which are associated with modulation of monoaminergic neurotransmission and/or regulation of the hypothalamic-pituitaryadrenal axis (Mason \& Pariante, 2006), are effective

* Address for correspondence: T. E. Schlaepfer, M.D., Department of Psychiatry/University Hospital, Sigmund-Freud-Strasse 25, 53105 Bonn, Germany.

(Email: schlaepf@jhmi.edu) in improving depressive symptoms in major depression (Mann, 2005). These medications, in conjunction with certain methods of psychotherapy and electroconvulsive therapy (ECT), are effective at alleviating depressive symptomatology in most patients (Andrews \& Nemeroff, 1994; Mann, 2005). However, these treatments do not work for all patients. Keller et al. (1992) studied the course of depression treatment prospectively over a 5-year period and found that the recovery rate decreased over time. 
Their findings of $9.3 \%(40 / 431$ patients) at the 5-year point supported earlier longitudinal research that found $6-13 \%$ of patients remained on a course of chronic unremitting depression despite adequate treatment. A more recent study found that $63.2 \%$ of patients included in the STAR-D study were not treated to remission during the acute study phase (Rush et al. 2006). Patients who do not respond to known treatment combinations including ECT are thus referred to as suffering from treatment-resistant depression (TRD).

A need for the development of alternative treatments for TRD that are effective, have fewer sideeffects or have longer-lasting antidepressant effects has been identified (Nestler, 1998; Schlaepfer \& Kosel, 2004). Vagus nerve stimulation (VNS) therapy is a type of treatment where a small electrical pulse is administered through an implanted neurostimulator to a bipolar lead attached to the left vagus nerve (George et al. 2000; Kosel \& Schlaepfer, 2003; Schlaepfer \& Kosel, 2004). This procedure has been studied in patients with treatment-resistant epilepsy and has been demonstrated to be effective in reducing seizure frequency (Ben-Menachem et al. 1994; The Vagus Nerve Stimulation Study Group, 1995; Handforth et al. 1998; Morris \& Mueller, 1999; Uthman, 2000 ; Ben-Menachem, 2001; Schachter, 2002). Significant and clinically meaningful antidepressant effects of VNS in epilepsy patients have been described, independent of reduction of seizure frequency (Elger et al. 2000; Harden et al. 2000; Helmstaedter et al. 2001).

The precise mechanism by which VNS might influence depressive symptoms is not known, but VNS clearly has effects on brain function (Kosel \& Schlaepfer, 2002; Groves \& Brown, 2005). Preliminary evidence for the mode of action of the putative antidepressant effect was obtained from brain imaging studies indicating that VNS affects the metabolism of limbic structures relevant to mood regulation (Henry et al. 1999). VNS has been shown to induce c-fos immunolabelling in several forebrain structures, including the posterior cortical amygdaloid nucleus, cingulate and retrosplenial cortex, ventromedial and arcuate hypothalamic nuclei (Naritoku et al. 1995). Another potential mechanism of action, supported by both animal and human studies, might be that VNS influences monoaminergic neurotransmission. Unlike other antidepressants, VNS seems not to be associated with an initial reduction in the firing rates of serotonergic neurones; in an animal study, raphe neurone firing rates increased progressively over 2 weeks, which could be an explanation for the slow and progressive increase of antidepressant response in clinical VNS studies (Dorr \& Debonnel, 2006). In a clinical VNS study, no significant change in cerebrospinal fluid (CSF) metabolites of norepinephrine (NE) and serotonin (5-HT) was seen in patients treated for 3 months compared with pretreatment levels while concentrations of homovanilinic acid in the CSF were increased significantly in treated patients compared to those treated with sham only (Carpenter et al. 2004). Reviews on the emerging body of functional neuroimaging [positron emission tomography (PET), single photon emission computed tomography (SPECT), and functional magnetic resonance imaging (fMRI)] effects of VNS have found the data difficult to reconcile, mainly because of the small sample sizes, different diagnoses, different types of concomitant antidepressant therapies and different time-point of scans obtained (Chae et al. 2003; Nemeroff et al. 2006).

A SPECT study in 12 patients with TRD found that after 4 weeks of VNS treatment, blood flow had decreased in the amygdala; hippocampus; thalamus; putamen; caudate; brainstem; subgenual, ventral anterior, posterior and dorsal anterior cingulate cortex; and orbito-, ventro- and dorsolateral prefrontal cortex. The only area of increased flow was found in the middle frontal gyrus (Zobel et al. 2005). In a ${ }^{15} \mathrm{O}-\mathrm{PET}$ study of four patients with VNS for 3 weeks, patients were scanned four times in an 'off-on' design. Blood flow increases were found in the orbitofrontal cortex, dorsal and ventral anterior cingulate; superior and inferior frontal gyri; cerebellum; and putamen; while decreases were found in temporal and parietal cortex (Conway et al. 2006).

Several studies assessing antidepressant properties of VNS in TRD have been conducted. The first open, unblinded four-centre pilot study (D01) of 60 patients showed efficacy in very treatment-resistant patients, of whom $30.5 \%$ met criteria for response after 3 months of VNS (Rush et al. 2000). The authors found that the number of unsuccessful adequate antidepressant treatment trials, rated by the Antidepressant Treatment History Form (ATHF; Prudic et al. 1990), during the current episode was inversely correlated with VNS response. The response rate was $50 \%$ in patients with two to three failed trials in the current episode, $29.1 \%$ after four to seven failed trials, and $0 \%$ after more than seven failed trials (Sackeim et al. 2001). The authors concluded that VNS is most effective in patients with moderate but not extreme levels of resistance to conventional antidepressant treatments.

A subsequent sham-controlled, multisite, doubleblind trial (D02) in a larger sample did not demonstrate superiority of active VNS treatment over sham treatment after 3 months. In the active VNS group $(n=112), 15.2 \%$ of the patients met criteria for response versus $10.0 \%$ in the sham group (Rush et al. 
2005a), despite excluding patients with more than six adequate antidepressant medication trials (as measured by the ATHF; Prudic et al. 1990). The authors suggested that the lack of superiority of active versus sham treatment could have been due to lower stimulation current. While in the first 3 months of VNS therapy output current in the D01 study ranged from 0.25 to $3.00 \mathrm{~mA}$ (mean $0.96 \pm 0.54 \mathrm{~mA}$ ) (Sackeim et al. 2001), in the D02 study output current ranged from 0.25 to $3.00 \mathrm{~mA}$ (mean $0.67 \pm 0.33 \mathrm{~mA}$ ) (Rush et al. 2005a). Longer-term outcomes following the first 3 months of VNS in the D02 study revealed that at 1 year of VNS therapy, $29.8 \%$ had responded and $17.1 \%$ had remitted (mean output current $1.0 \mathrm{~mA}$, range $0-2 \mathrm{~mA}$ ).

The results of 1 year of VNS in the D02 study were significantly superior to outcomes at 1 year in a cluster-matched but more randomized comparison sample of patients receiving treatment as usual (TAU) (George et al. 2005). Response rates as measured by the 24-item Hamilton Depression Rating Scale (HAMD-24) were $29.8 \%$ (D02) and $12.5 \%$ (TAU, $n=104)$ at 1 year. This TAU sample was acquired initially to define prospectively the outcome of TAU on such patients. In both the long-term TAU and VNS samples, medications and psychotherapy could be added or dropped and doses could be changed, and other non-pharmacological treatments [ECT, transcranial magnetic stimulation (TMS), light therapy] could be used. A careful examination of the potential contributions to the differential outcomes in these two samples failed to reveal that any baseline covariate, intercurrent treatment or medication management differences could account for the difference in outcome. In summary, results of open-label, uncontrolled trials examining efficacy of VNS in treatmentresistant major depression seem to point to both acute and longer-term effectiveness.

The European study of VNS for TRD reported here (D03) was conducted to determine if the USA results could be replicated using a similar study protocol in a different patient population with different severity and in a different health-care environment. We report on the acute and medium-term outcome after 3 and 12 months in a European patient sample.

\section{Method \\ Protocols}

Patients with treatment-resistant major depression participating in the D03 study were enrolled from 2001 to 2005 in six European countries (Belgium, Germany, Ireland, Sweden, Switzerland, the UK) in an uncontrolled open-label study design. This protocol was very similar to the D01 study conducted in the USA, except that (1) study inclusion required a score $\geqslant 20$ on the HAMD-24 (Hamilton, 1967) in the D03 study, as opposed to $\geqslant 20$ on the 28-item HAMD (HAMD-28) in the D01 study, (2) the maximum age at entry was 80 in the D03 study and 70 in the D01 study, and (3) the number of failed adequate medication trials was $\geqslant 2$ but $<6$ in the D03 study versus $\geqslant 2$ in the D01 study.

\section{Patients}

Patients suffered from non-psychotic major depressive disorder (MDD) or bipolar I or II disorder (according to DSM-IV diagnosis). The current major depressive episode (MDE) had lasted more than 2 years and/or the patient had had more than four lifetime MDEs. At study entry the patients ranged in age from 18 to 80 years. Pregnant women and those not using generally accepted methods of birth control were excluded. Patients had to have experienced inadequate antidepressant response with 2-6 treatments during the current MDE. Treatment adequacy was rated with the Antidepressant Treatment History Form (ATHF), an adequate trial resulted in an Antidepressant Resistance Rating (ARR) score of $\geqslant 3$ (Prudic et al. 1990). Patients with bipolar disorder had to be either treatment resistant to, intolerant to, or have a medical contraindication to, lithium. All patients had to have had at least 6 weeks of psychotherapy (during any MDE) that resulted in inadequate clinical improvement.

Patients with atypical or psychotic depression were excluded, as were patients with a history of schizophrenia, schizo-affective disorder or delusional disorder, bipolar disorder with rapid cycling and patients with a secondary diagnosis of delirium, dementia, amnesic or other cognitive disorder. Patients with clinically significant, current suicidal ideation and those with health risks related to the surgical procedures and stimulation were also excluded.

\section{Study overview}

The study was an open, unblinded, not sham controlled, multi-centre trial conducted at nine European sites. The ethics committee at each study site approved the study protocol. After written informed consent was obtained, patients completed a baseline period (up to 4 weeks) before undergoing device implantation. Patients were assessed at two study visits during this period. Only patients with a score of 20 or higher on the HAMD-24 at both visits were implanted. After implantation, a 2-week single-blinded 
recovery period followed. Patients were told 'stimulation may or may not be turned on immediately after implantation'. Stimulation was initiated if patients scored 18 or more on the HAMD-24 at the end of the recovery period. During the following 2-week stimulation adjustment period, stimulation was increased individually to the maximal, comfortably tolerated level. Stimulation parameters were then set and remained fixed for the following 8 weeks of the acute study period. During the acute study period, clinic visits were held at weeks 1, 2, 3, 4, 6 and 8 . Following the acute study period ( 3 months after implantation), patients entered the long-term followup period. If patients met criteria for response at the end of the acute study period (reduction of $\geqslant 50 \%$ of the baseline HAMD-24 score), monthly visits followed for the remaining 9 months; if they did not meet the response criteria, quarterly visits followed for the remaining 9 months. During the long-term followup period, changes in stimulation parameters and medications were permitted.

\section{VNS therapy: implantation and treatment}

Implantation and treatment parameters used in this study were identical to those used in epilepsy studies (Ben-Menachem et al. 1994; Handforth et al. 1998). The VNS therapy system (Cyberonics Inc., Houston, TX, USA) consisted basically of three parts: (1) the implantable, multi-programmable bipolar pulse generator, which is similar to a cardiac pacemaker in its size and shape; (2) two helicoidal electrodes that are wrapped around the vagus nerve and are linked to the pulse generator by a bipolar lead; and (3) a programming wand linked to a computer running a programming software, which allows non-invasive programming, functional assessment (device diagnostics) and data retrieval. The pulse generator is implanted in a subcutaneous pocket in the left chest wall and the electrodes are attached to the vagus nerve. The electrodes are connected to the stimulator through a subcutaneous tunnel. After the recovery period, stimulation was initiated at the following parameters: current intensity of $0.25 \mathrm{~mA}$, pulse frequency of $20 \mathrm{~Hz}$, pulse width of $500 \mu \mathrm{s}$ with stimulation on for $30 \mathrm{~s}$ and off for $5 \mathrm{~min}$. The output current was then (during the stimulation adjustment period) increased in $0.25 \mathrm{~mA}$, increments until an individual maximal tolerable and comfortable level was reached. At each study visit, the accuracy of the stimulation parameters was verified.

\section{Concomitant therapy}

Concomitant treatment with antidepressant medications (antidepressants, mood stabilizers or other psychotropic medications) was permitted, but it had to be stable for 4 weeks prior to study entry (baseline), during the recovery period and the acute study phase (i.e. for the first 12 weeks following implantation). Thereafter, treatments could be added, adjusted or stopped. Investigational drugs and treatment with another investigational device were not permitted. Other non-psychiatric medications (e.g. antibiotics, analgesics) were allowed and were recorded at each visit.

\section{Evaluation/outcome measures}

Outcome parameters. Baseline depression severity (HAMD-24 score at baseline) was compared to ratings 2 weeks after implantation (end of recovery period); after 3 months of VNS (end of acute study period); and after an additional 3, 6 and 9 months (end of long-term study period).

Primary clinical outcome. Response was defined as a $\geqslant 50 \%$ reduction in HAMD-24 score from the baseline period (mean of visits 1 and 2), remission was defined as a HAMD-24 score $\leqslant 10$.

Secondary clinical outcome parameters. Secondary outcomes were assessed on the Montgomery-Asberg Depression Rating Scale (MADRS) and the Inventory of Depressive Symptomatology Self-Rated (IDS-SR).

\section{Adverse events (AEs)}

AEs events were collected by the COSTART (Coding System for Thesaurus of Adverse Reaction Terms) (FDA, 1995). AEs were defined as events occurring on or after the date of implantation, events not reported as signs or symptoms at baseline and/or worsening in severity or frequency. Presence of mania was monitored using the Young Mania Rating Scale (YMRS); a score of $\geqslant 15$ was used as threshold for the diagnosis of mania.

\section{Statistical analysis}

Baseline demographic and clinical characteristics of the D03 and D01 patients were compared by using the $t$ test or the Wilcoxon rank sum test for continuous measures, and $\chi^{2}$ or Fisher's exact tests for categorical measures. Changes in HAMD scores were analysed with repeated-measures analysis of variance [SPSS repeated generalized linear model (repGLM)]. Analyses are based on observed cases (OCs) and last observation carried forward (LOCF), as indicated. Multiple comparisons were corrected using the Bonferroni method. 


\section{Results}

\section{Enrolment}

A total of 84 patients were eligible for inclusion in the study and gave their signed informed consent. Ten patients withdrew consent before implantation. Of the 74 implanted patients, four withdrew consent and discontinued study participation during the acute study period. Seven patients dropped out during the first-year long-term study period, five of them withdrew consent due to AEs or lack of efficacy (two were explanted), and two patients committed suicide. Demographic and baseline characteristic data for all 74 implanted patients were analysed; outcome data for 70 acute study period completers were available. Long-term data for 61 patients ( 6 months), 55 patients (9 months) and 60 patients (1 year) were available. Various assessments are missing because of not-conducted visits. Baseline characteristics of both D01 and D03 patient populations are summarized in Table 1. This table shows that unsuccessful mood disorder treatments during the current MDE averaged $6.2 \pm 3.1$. Of these, $4 \pm 2.4$ were classic antidepressant treatments, whereas $3.5 \pm 1.3$ trails met ATHF criteria for adequacy. During the current episode, $38 \%(28 / 74)$ of patients had received ECT. The baseline scores of depression scales (HAMD-24, MADRS, IDS-SR) are consistent with a severe level of depression.

\section{Stimulation parameters}

Most patients $(86 \%)$ were stimulated with a $20 \mathrm{~Hz}$ frequency and a $500 \mu$ s pulse width for $30 \mathrm{~s}$ on and $5 \mathrm{~min}$ off. The others differed in the following parameter settings: one patient had a frequency of $30 \mathrm{~Hz}$ and a pulse width of $500 \mu \mathrm{s}$, seven patients had a frequency of $20 \mathrm{~Hz}$ and a pulse width of $250 \mu \mathrm{s}$, and one patient each had a frequency of $15 \mathrm{~Hz}$ and $10 \mathrm{~Hz}$ and a pulse width of $250 \mu \mathrm{s}$; usually parameters were changed to increase tolerability. The output currents ranged from 0.25 to $2 \mathrm{~mA}$, (mean $1.2 \pm 0.34 \mathrm{~mA}$, median $1.25 \mathrm{~mA})$. During the long-term follow-up period the median output current was $1.25 \mathrm{~mA}$, (range $0.25-2.25 \mathrm{~mA})$.

\section{Efficacy}

Primary clinical outcome measures

The severity of depression as measured by the HAMD-24 diminished significantly after 3, 6, 9 and 12 months of VNS compared to baseline severity of depression [repGLM, $F(4)=30.028, p=0.000$ ]. Analysis under LOCF conditions demonstrated that the decrease in the severity of depression at every time-point was significant [repGLM, $F(4)=30.718, p=0.000]$. The percentage of the patient population reaching the response criterion was $36 \%(25 / 70)$ after 3 months, increasing to $44 \%$ after 6 months (27/61), 53\% (29/55) after 9 months and 55\% (33/60) after 1 year of VNS. Under LOCF conditions, rates of response reached $34 \%(25 / 74)$ after 3 months, 39\% (29/74) after 6 months, $46 \%(34 / 74)$ after 9 months and 47\% (35/74) after 1 year of VNS.

\section{Secondary clinical outcome measures, MADRS and IDS-SR}

Reduction in severity of depression measured by MADRS and the IDS-SR was also significant. Decrease in the MADRS score reached significance at every key outcome point compared to baseline score in both samples [repGLM, $F(4)=34.613, p=0.000]$. The score on the self-rating questionnaire (IDS-SR) also decreased significantly [repGLM, $F(4)=23.256, p=0.000$ ] and steadily over time. The mean percentage of decrease reached $41 \%$ after 1 year.

\section{Comparison of results of D01 and D03 study}

Depression severity rating: HAMD-28

Primary outcome measure in the D03 study was HAMD-24, but HAMD-28 has also been assessed and is used here for comparison. The severity of depression as measured by the HAMD-28 diminished significantly under VNS (Fig. 1) The decreases after 3, 6,9 and 12 months compared to baseline score reached significance in both samples [D03 repGLM, $F(4)=$ 37.880, $p=0.000$; D01 repGLM, $F(4)=31.255, p=0.000$, observed cases]. Analysis under LOCF conditions confirmed these results [D03 repGLM, $F(4)=41.628$, $p=0.000$; D01 repGLM, $F(4)=36.455, p=0.000]$. The decrease was larger in the D03 sample but the differences did not reach significance at any time-point.

\section{Rates of response and remission}

Figure 2 shows rates of response and remission in the first year of VNS therapy. Response was defined as a reduction of $\geqslant 50 \%$ in the HAMD-28 score, remission as a HAMD-28 score $<10$. Response and remission rate increased steadily over time in both samples; response rate reached $53 \%$ after 12 months; remission rate reached $33 \%$.

\section{Pattern of response analysis}

In order to evaluate characteristics of response during the first year of VNS, the sample was assigned to four groups regarding onset and proceed of response: (1) no response, (2) fluctuating response, (3) early 
Table 1. Demographic and clinical characteristics of the D03 and D01 samples

\begin{tabular}{|c|c|c|c|}
\hline & $\begin{array}{l}\text { D03 } \\
(n=74)\end{array}$ & $\begin{array}{l}\text { D01 } \\
(n=60)\end{array}$ & $p$ \\
\hline Age at implant (years) & $47.4 \pm 11.7$ & $46.8 \pm 8.7$ & $0.719^{\mathrm{b}}$ \\
\hline Gender, female & $50(67.6)$ & $39(65)$ & $0.754^{\mathrm{a}}$ \\
\hline \multicolumn{4}{|l|}{ DSM-IV diagnosis } \\
\hline Unipolar, recurrent & $41(55.4)$ & $28(47)$ & \multirow[t]{4}{*}{$0.009^{\mathrm{a}}$} \\
\hline Unipolar, single episode & $13(17.5)$ & $16(27)$ & \\
\hline Bipolar I & $9(12.2)$ & $6(10)$ & \\
\hline Bipolar II & $11(14.9)$ & $10(17)$ & \\
\hline Total unipolar & $54(73)$ & $44(73)$ & $0.963^{\mathrm{a}}$ \\
\hline Total length of affective disorder (years) & $18.54 \pm 9.9$ & $18.1 \pm 10.9$ & $0.811^{\mathrm{b}}$ \\
\hline Length of current episode (years ) & $3.46 \pm 6.25$ & $9.9 \pm 10.8$ & $0.000^{\mathrm{b}}$ \\
\hline \multicolumn{4}{|l|}{ Number of depressive episodes lifetime } \\
\hline $0-2$ & $17(23)$ & $35(58)$ & \multirow[t]{5}{*}{$0.000^{\mathrm{a}}$} \\
\hline $3-5$ & $20(27)$ & $18(30)$ & \\
\hline $6-10$ & $19(2628)$ & $3(5)$ & \\
\hline$>10$ & $16(2124)$ & $4(7)$ & \\
\hline Unknown & $2(3)$ & - & \\
\hline Total mood disorder treatments & $6.2 \pm 3.1$ & $15.7 \pm 7.9$ & $0.000^{\mathrm{b}}$ \\
\hline Antidepressants & $4 \pm 2.4$ & $8.6 \pm 4.0$ & $0.000^{\mathrm{b}}$ \\
\hline Other mood disorder treatments & $1.6 \pm 1.4$ & $4.8 \pm 3.5$ & $0.000^{\mathrm{b}}$ \\
\hline Anxiolytics & $0.7 \pm 0.8$ & $1.9 \pm 1.4$ & $0.000^{\mathrm{b}}$ \\
\hline Neuroleptics & $0.0 \pm 0.0$ & $0.5 \pm 0.9$ & $0.000^{\mathrm{b}}$ \\
\hline \multicolumn{4}{|l|}{ ATHF adequacy rating } \\
\hline $\begin{array}{l}\text { Unsuccessful adequate medication trials in } \\
\text { the current major depressive episode }\end{array}$ & $3.5 \pm 1.3$ & $4.8 \pm 2.7$ & $0.000^{\mathrm{a}}$ \\
\hline ECT in current episode & $28(38)$ & $34(57)$ & $0.030^{\mathrm{a}}$ \\
\hline ECT lifetime & $37(50)$ & $40(67)$ & $0.052^{\mathrm{a}}$ \\
\hline HAMD-24 & $28.6 \pm 5.3$ & - & - \\
\hline HAMD-28 & $34.0 \pm 5.8$ & $36.8 \pm 5.8$ & $0.006^{\mathrm{b}}$ \\
\hline MADRS & $32.9 \pm 6.4$ & $33.4 \pm 5.7$ & $0.635^{\mathrm{b}}$ \\
\hline IDS-SR & $47.3 \pm 9.6$ & - & - \\
\hline
\end{tabular}

ATHF, Antidepressant Treatment History Form; ECT, electroconvulsive therapy; HAMD, Hamilton Depression Rating Scale; MADRS, Montgomery-Asberg Depression Rating Scale; IDS-SR, Inventory of Depressive Symptomatology Self-Rated; S.D., standard deviation.

Values are given as $n(\%)$ or mean \pm S.D.

Values in bold indicate statistical significant differences between studies.

Samples were comparable on most baseline characteristics; there was no difference in age, gender and lifetime psychiatric illness. In terms of number of prior episodes, duration of current episode and overall number of mood disorder treatments in the current episode, the patient populations were different. In addition, the number of adequate antidepressant trials and the number of patients receiving ECT in the current episode were significantly higher in the D01 sample $\left[\chi^{2}(1)=4.725, p=0.030\right]$. Baseline scores of depression scales (HAMD, MADRS, IDS-SR) indicated a severe level of depressive symptoms in both samples. The difference in the baseline HAMD-28 score between the two samples reached significance [ANOVA, $F(1)=7.805, p=0.006]$ but not the difference in baseline MADRS score [ANOVA, $F(1)=0.226, p=0.635]$. The scores of the IDS-SR are only available for the D03 sample.

${ }^{\text {a }} \chi^{2}$ test.

${ }^{\mathrm{b}}$ ANOVA $F$. 


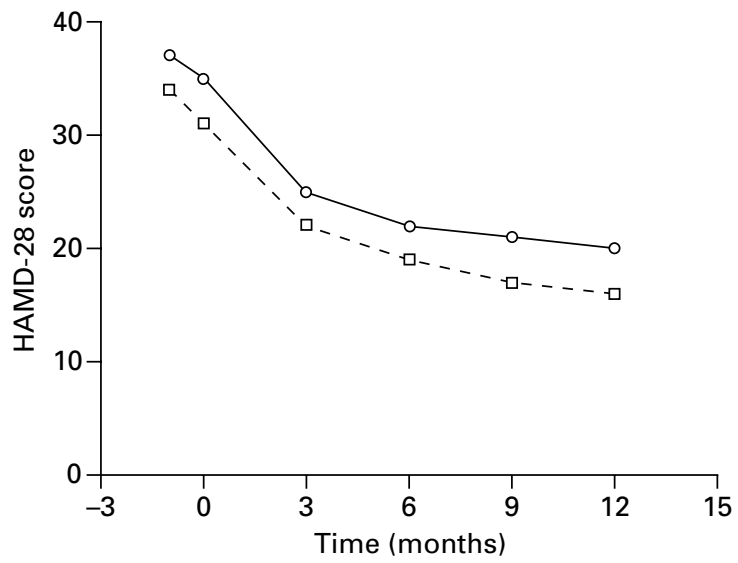

Fig. 1. Mean scores on the 28-item Hamilton Depression Rating Scale (HAMD-28) at study visits for the D03 (-- $\square--)$ and the D01 (-○—) study. Severity of depression as assessed by the HAMD-28 score diminished significantly under vagus nerve stimulation (VNS) : the decreases after 3, 6, 9 and 12 months compared to the baseline ( -1 month) score reached significance in both study samples. The decrease was significantly larger after 1 year of VNS.

response and (4) late response. Patients in the 'no response' group did not meet criteria for response at any key outcome point, in the 'fluctuating response' group patients met criteria for response only once or twice but relapsed, in the 'early response' group patients met criteria for response after 3 months of VNS therapy and remained responders for the rest of the year, and in the 'late response' group patients met criteria for response after 6 or 9 months and remained responders for the rest of the year. Patients in the early and late response group can therefore be counted among sustained responders. In the D03 sample only 1 -year completers were considered $(n=59)$. The percentage of sustained responders is higher in the D03 sample, with $44 \%$ of the patients in the D03 sample showing a sustained response compared to $32 \%$ in the D01 sample. The percentages of patients with a fluctuating response were almost equal (32\% in the D03 and $36 \%$ in the D01), but the percentage of patients never meeting criteria for response in the first year was $24 \%$ in the D03 sample compared to $32 \%$ in the D01 sample.

\section{Adverse events (AEs)}

Table 2 summarizes the rates of AEs during the first year of VNS and displays them in comparison to published safety data from the D02 pivotal studies on VNS in depression (Rush et al. 2005b). The most common side-effects in the acute study period were voice alteration $(63 \%)$, cough $(26 \%)$, pain $(20 \%)$ and dyspnoea (10\%). After 1 year of stimulation the most
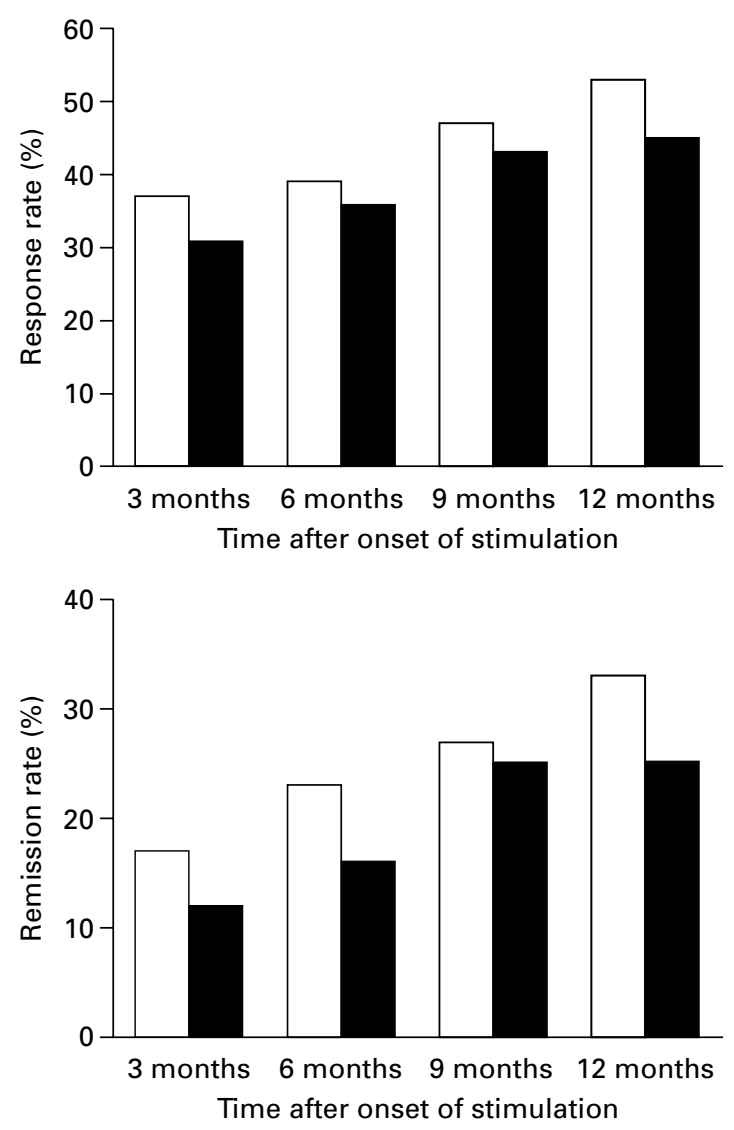

Fig. 2. Response and remission rates (\%) in the D03 ( $\square$ ) and D01 (ם) samples, observed cases. Response was defined as reduction of $\geqslant 50 \%$ in the 28-item Hamilton Depression Rating Scale (HAMD-28) score compared to baseline HAMD28 score; remission was defined as a HAMD-28 score of $\leqslant 10 \%$. Rates of both response and remission in the D03 sample exceeded rates in the D01 sample, although differences did not reach statistical significance.

common side-effects were voice alteration (55\%) and dyspnoea $(10 \%)$. The side-effects were typically restricted to the time of stimulation, and mild sideeffects were classified as moderate diminished typically over time. No patient discontinued study participation due to AEs. During the first year, nine patients withdrew consent, four during the acute study period and seven during the long-term study period (two of them were explanted due to lack of effectiveness). There were 15 serious AEs reported during the first year of VNS, resulting in hospitalizations: seven episodes of worsening of depression, two committed suicides, one brain haemorrhage due to suicide attempt, one episode of nephrolithiasis, one of cholelithiasis, one of pulmonary embolism, one of mania and one of syncope. Of these 15 serious AEs, only manic episodes were judged by the investigator as being possibly related to stimulation. 
Table 2. Adverse events recorded in the D03 study compared to published USA data (Rush et al. 2005b)

\begin{tabular}{|c|c|c|c|c|c|c|c|c|}
\hline \multirow[b]{2}{*}{ Adverse events } & \multicolumn{2}{|c|}{3 months } & \multicolumn{2}{|c|}{6 months } & \multicolumn{2}{|c|}{9 months } & \multicolumn{2}{|c|}{12 months } \\
\hline & $\begin{array}{l}\text { D03 } \\
(n=70)\end{array}$ & $\begin{array}{l}\text { USA } \\
(n=232)\end{array}$ & $\begin{array}{l}\text { D03 } \\
(n=61)\end{array}$ & $\begin{array}{l}\text { USA } \\
(n=225)\end{array}$ & $\begin{array}{l}\mathrm{D} 03 \\
(n=54)\end{array}$ & $\begin{array}{l}\text { USA } \\
(n=218)\end{array}$ & $\begin{array}{l}\text { D03 } \\
(n=60)\end{array}$ & $\begin{array}{l}\text { USA } \\
(n=209)\end{array}$ \\
\hline Suicide attempts & - & 1 & 1 & 1 & - & 1 & - & 1 \\
\hline Suicide & - & - & 2 & - & - & - & - & - \\
\hline Voice alteration & 63 & 58 & 2 & 60 & 2 & 57 & 2 & 54 \\
\hline Worsening depression & 1 & 5 & - & 7 & 2 & 5 & - & 6 \\
\hline Cough increased & 26 & 24 & 3 & 9 & 2 & 7 & 3 & 6 \\
\hline Dyspnoea & 10 & 14 & 5 & 16 & 8 & 15 & 10 & 16 \\
\hline Pain & 20 & 6 & - & 6 & 5 & 5 & - & 6 \\
\hline Pharyngitis & 6 & 6 & 3 & 4 & 2 & 4 & 3 & 5 \\
\hline Headache & 3 & 5 & 2 & 4 & 2 & 4 & 2 & 4 \\
\hline Device site pain & 4 & N.A. & - & N.A. & - & N.A. & - & N.A. \\
\hline Pain neck & 7 & N.A. & 3 & N.A. & - & N.A. & - & N.A. \\
\hline Pain ear & 7 & N.A. & 2 & N.A. & - & N.A. & - & N.A. \\
\hline Neuralgia & 3 & N.A. & 56 & N.A. & 54 & N.A. & 55 & N.A. \\
\hline Twitching & 1 & N.A. & - & N.A. & - & N.A. & - & N.A. \\
\hline Manic reaction & 1 & 1 & - & $<1$ & - & - & 2 & - \\
\hline Paresthesia & 1 & 11 & - & 7 & 2 & 3 & - & 4 \\
\hline Dyspepsia & 1 & N.A. & 2 & N.A. & 2 & N.A. & 2 & N.A. \\
\hline Dysphagia & 6 & 13 & - & 8 & - & 7 & 2 & 4 \\
\hline Reflux & 1 & N.A. & 2 & N.A. & - & N.A. & - & N.A. \\
\hline Gingivitis & 1 & N.A. & - & N.A. & - & N.A. & - & N.A. \\
\hline Nausea & 1 & 6 & 2 & 2 & - & 2 & 2 & 2 \\
\hline Laryngitis & - & N.A. & 1 & N.A. & - & N.A. & - & N.A. \\
\hline Hypertonia & - & N.A. & - & N.A. & - & N.A. & 2 & N.A. \\
\hline Syncope & - & N.A. & 2 & N.A. & - & N.A. & - & N.A. \\
\hline Angina pectoris & - & N.A. & 2 & N.A. & - & N.A. & - & N.A. \\
\hline Pruritis & 4 & N.A. & - & N.A. & - & N.A. & - & N.A. \\
\hline
\end{tabular}

Adverse events are possibly, probably, or definitely related to stimulation based on the observed cases.

Values given in the table are percentages.

\section{Discussion}

In this study we report on effectiveness data of the European (D03) multi-centre VNS study, and compare them to the effectiveness data of the USA (D01) (Rush et al. 2000) study. Both studies were similar in protocol design and size, but patient samples were different regarding baseline characteristics, for example the proportion of bipolar I and II diagnoses, length of current episode, number of current episodes, total of mood disorder treatments, degree of treatment resistance, baseline depression scores and number of previous ECT treatments. In both studies repeated ANOVA showed significant reduction in severity of depression (as measured by HAMD-24, HAMD-28 and MADRS) over time. The reduction was larger in the European sample: response rates reached 37\% after 3 months and increased to $53 \%$ after 1 year of VNS therapy. Remission rates reached $17 \%$ after
3 months and increased to $33 \%$ after 1 year of VNS therapy. Rates of response and remission in the European sample increased steadily over time, as in the published D01 results. In our study, $44 \%$ of the patients showed sustained response, defined by the absence of relapse after onset of response during the first year of VNS. The higher efficacy in this study compared to the previously published one can probably be attributed to the lower measures of baseline depressivity.

A major shortcoming of this study, as for the USA (D01) study, is the fact that effectiveness was not assessed in a sham controlled design, limiting interpretations on clinical utility. It has been argued that a controlled design would be unethical, because eligible patients would be too depressed to be taken off their medications and given only sham stimulation (Shuchman, 2007). While this is correct in principle, it would certainly be scientifically important to plan 


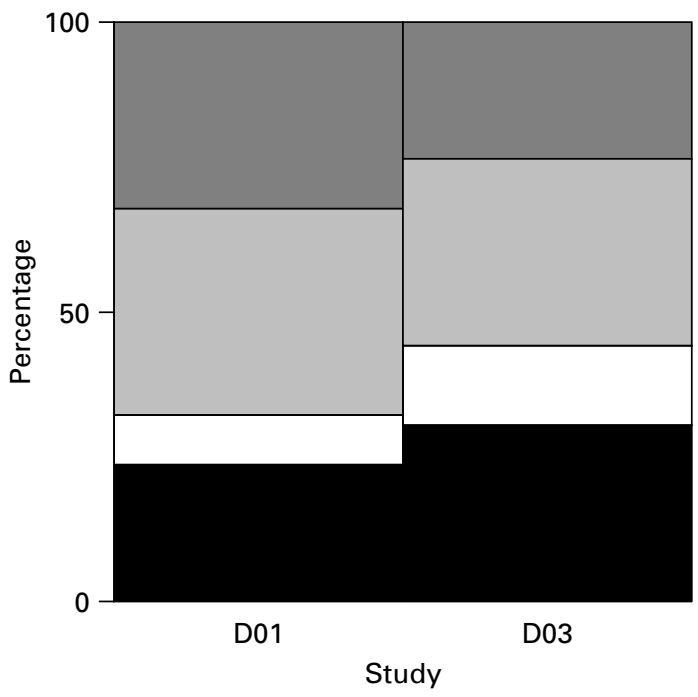

Fig. 3. Proportion of patients meeting different criteria for pattern of response to vagus nerve stimulation (VNS) therapy. The percentage of sustained responders was higher in the D03 sample than in the D01 sample (HAMD-28, observed cases). The percentages of patients with fluctuating response were almost equal, but the percentage of patients never meeting criteria for response in the first year was lower in the D03 sample compared to the D01 sample. Early responders ( $\mathbf{\square})$ : response criteria first achieved after 3 months, continuous to 12 months. Late responders $(\square)$ : response criteria first achieved after 6 or 9 months and continuous to 12 months. Fluctuating responders $(\square)$ : response criteria achieved once/twice after 3, 6, 9 or 12 months, no continuous response. Non-responders $(\square)$ : no response at any key outcome point.

future VNS protocols in a controlled way, as VNS is studied as an add-on to existing antidepressant medication. In addition, it might be argued that because VNS is an invasive procedure involving surgical intervention, implantation of a device and repetitive slight discomfort and voice alteration ( $30 \mathrm{~s}$ of stimulation every $5 \mathrm{~min}$ ), it is associated with an even greater placebo response than those detected in drug trials. In future trials of VNS for depression, it might therefore be valuable to study the specific characteristics of personality of a patient population with treatment resistance interested in this procedure to judge whether personality features contribute differentially to treatment effects. Although we have no general indication in the patients studied in the D03 trial, it might well be that these patients have a higher degree of axis 2 co-morbidity, possibly conferring a different placebo response.

However, there are some factors that make it unlikely that the observed antidepressant response in this study can be attributed to placebo effects alone. First, the patients studied all suffered from severe TRD, and such patients are known to be less likely to show placebo response (Schatzberg \& Kraemer, 2000). Regarding the treatment of depression in an elderly patient group, high placebo response rates are seen particularly with milder depression, but more effectiveness is noted with higher drug-placebo differences in trials with more severe forms of depression (Schatzberg \& Kraemer, 2000; Lyketsos et al. 2003).

Second, response rates in a comparable sample of patients with severe TRD reached $5.8 \%$ after 3 months and $11.6 \%$ after 12 months of treatment as usual, indicating a very low likelihood of sustained treatment response despite receiving a variety of treatments consisting of various classes of antidepressants with augmentation including combination strategies, psychotherapy and ECT (Dunner et al. 2006).

Third, we demonstrated a high proportion of sustained antidepressant response over the time of observation (see Fig. 3). In antidepressant trials, placebo response appears to be less stable than the improvement attributable to drugs (Dago \& Quitkin, 1995). This has been demonstrated, for example, in a 6-week placebo-controlled study; patients who had responded were randomized either to continue on the same dose of citalopram or to receive placebo for a further period of 24 weeks. Patients who switched to placebo had re-emergence of their depressive symptoms at a significantly higher rate than patients maintained on citalopram (Montgomery et al. 1993).

In summary, our results seem to point to antidepressant properties of VNS in a very treatmentresistant patient population, and even if these are due to limitations in the protocol, the putative contribution of the placebo effect cannot be assessed.

\section{Acknowledgements}

We are indebted to A. John Rush, University of Texas Southwestern Medical Center in Houston, for a critical review of this paper and valuable suggestions for the analysis.

\section{Declaration of Interest}

The authors have performed this study and the analysis of its results; the conduct of the study was partly funded by the manufacturer of the (VNS) Therapy $^{\mathrm{TM}}$ System, Cyberonics Inc., Houston, TX, USA. The amount of funding covered in the case of the Bern study centre was $16 \%$ of the total cost. The sponsor had no influence whatsoever on the analysis, writing or editing or on the final decision to submit the manuscript. 


\section{References}

Andrews JM, Nemeroff CB (1994). Contemporary management of depression. American Journal of Medicine 97, 24S-32S.

Ben-Menachem E (2001). Vagus nerve stimulation, side effects, and long-term safety. Journal of Clinical Neurophysiology 18, 415-418.

Ben-Menachem E, Manon-Espaillat R, Ristanovic R, Wilder BJ, Stefan H, Mirza W, Tarver WB, Wernicke JF (1994). Vagus nerve stimulation for treatment of partial seizures: 1. A controlled study of effect on seizures. First International Vagus Nerve Stimulation Study Group. Epilepsia 35, 616-626.

Carpenter LL, Moreno FA, Kling MA, Anderson GM, Regenold WT, Labiner DM, Price LH (2004). Effect of vagus nerve stimulation on cerebrospinal fluid monoamine metabolites, norepinephrine, and gamma-aminobutyric acid concentrations in depressed patients. Biological Psychiatry 56, 418-426.

Chae JH, Nahas Z, Lomarev M, Denslow S, Lorberbaum JP, Bohning DE, George MS (2003). A review of functional neuroimaging studies of vagus nerve stimulation (VNS). Journal of Psychiatric Research 37, 443-455.

Conway CR, Sheline YI, Chibnall JT, George MS, Fletcher JW, Mintun MA (2006). Cerebral blood flow changes during vagus nerve stimulation for depression. Psychiatry Research 146, 179-184.

Dago P, Quitkin F (1995). Role of the placebo response in the treatment of depressive disorders. CNS Drugs 4, 335-340.

Dorr AE, Debonnel G (2006). Effect of vagus nerve stimulation on serotonergic and noradrenergic transmission. Journal of Pharmacology and Experimental Therapeutics 318, 890-898.

Dunner D, Rush A, Russell J, Burke M, Woodard S, Wingard P, Allen J (2006). Prospective, long-term, multicenter study of the naturalistic outcomes of patients with treatment-resistant depression. Journal of Clinical Psychiatry 67, 688-695.

Elger G, Hoppe C, Falkai P, Rush A, Elger C (2000). Vagus nerve stimulation is associated with mood improvements in epilepsy patients. Epilepsy Research 42, 203-210.

FDA (1995). COSTART: Coding Symbols for Thesaurus of Adverse Reaction Terms. US Department of Health and Human Services, Center for Drug Evaluation and Research, Food and Drug Administration, Center for Drugs and Biologics: Rockville, MD.

George MS, Rush AJ, Marangell LB, Sackeim HA, Brannan SK, Davis SM, Howland R, Kling MA, Moreno F, Rittberg B, Dunner D, Schwartz T, Carpenter L, Burke M, Ninan P, Goodnick P (2005). A one-year comparison of vagus nerve stimulation with treatment as usual for treatment-resistant depression. Biological Psychiatry 58, 364-373.

George MS, Sackeim HA, Rush AJ, Marangell LB, Nahas Z, Husain MM, Lisanby S, Burt T, Goldman J, Ballenger JC (2000). Vagus nerve stimulation: a new tool for brain research and therapy. Biological Psychiatry 47, 287-295.

Groves DA, Brown VJ (2005). Vagal nerve stimulation: a review of its applications and potential mechanisms that mediate its clinical effects. Neuroscience and Biobehavioral Reviews 29, 493-500.

Hamilton M (1967). Development of a rating scale for primary depressive illness. British Journal of Social and Clinical Psychology 6, 278-296.

Handforth A, DeGiorgio C, Schachter S, Uthman B, Naritoku D, Tecoma E (1998). Vagus nerve stimulation therapy for partial-onset seizures: a randomized active-control trial. Neurology 51, 48-55.

Harden CL, Pulver MC, Ravdin LD, Nikolov B, Halper JP, Labar DR (2000). A pilot study of mood in epilepsy patients treated with vagus nerve stimulation. Epilepsy and Behavior 1, 93-99.

Helmstaedter C, Hoppe C, Elger CE (2001). Memory alterations during acute high-intensity vagus nerve stimulation. Epilepsy Research 47, 37-42.

Henry TR, Votaw JR, Pennell PB, Epstein CM, Bakay RAE, Faber TL, Grafton ST, Hoffman JM (1999). Acute blood flow changes and efficacy of vagus nerve stimulation in partial epilepsy. Neurology 52, 1166-1173.

Keller MB, Lavori PW, Mueller TI, Endicott J, Coryell W, Hirschfeld RM, Shea T (1992). Time to recovery, chronicity, and levels of psychopathology in major depression. A 5-year prospective follow-up of 431 subjects. Archives of General Psychiatry 49, 809-816.

Kosel M, Schlaepfer TE (2002). Mechanisms and state of the art of vagus nerve stimulation. Journal of ECT 18, 189-192.

Kosel M, Schlaepfer TE (2003). Beyond the treatment of epilepsy: new applications of vagus nerve stimulation (VNS) in psychiatry. CNS Spectrums 8, 515-521.

Lyketsos CG, DelCampo L, Steinberg M, Miles Q, Steele CD, Munro C, Baker AS, Sheppard JM, Frangakis C, Brandt J, Rabins PV (2003). Treating depression in Alzheimer disease: efficacy and safety of sertraline therapy, and the benefits of depression reduction: the DIADS. Archives of General Psychiatry 60, 737-746.

Mann JJ (2005). The medical management of depression. New England Journal of Medicine 353, 1819-1834.

Mason BL, Pariante CM (2006). The effects of antidepressants on the hypothalamic-pituitary-adrenal axis. Drug News and Perspectives 19, 603-608.

Montgomery SA, Rasmussen JG, Tanghoj P (1993). A 24-week study of $20 \mathrm{mg}$ citalopram, $40 \mathrm{mg}$ citalopram, and placebo in the prevention of relapse of major depression. International Clinical Psychopharmacology 8, 181-188.

Morris 3rd GL, Mueller WM (1999). Long-term treatment with vagus nerve stimulation in patients with refractory epilepsy. The Vagus Nerve Stimulation Study Group E01-E05. Neurology 53, 1731-1735.

Naritoku DK, Terry WJ, Helfert RH (1995). Regional induction of fos immunoreactivity in the brain by anticonvulsant stimulation of the vagus nerve. Epilepsy Research 22, 53-62.

Nemeroff CB, Mayberg HS, Krahl SE, McNamara J, Frazer A, Henry TR, George MS, Charney DS, Brannan SK (2006). VNS therapy in treatment-resistant depression: clinical evidence and putative neurobiological mechanisms. Neuropsychopharmacology 31, 1345-1355.

Nestler E (1998). Antidepressant treatments in the 21st century. Biological Psychiatry 44, 526-533. 
Prudic J, Sackeim HA, Devanand DP (1990). Medication resistance and clinical response to electroconvulsive therapy. Psychiatry Research 31, 287-296.

Rush AJ, George MS, Sackeim HA, Marangell LB, Husain MM, Giller C, Nahas Z, Haines S, Simpson RK, Goodmann R (2000). Vagus nerve stimulation (VNS) for treatment-resistant depression: a multicenter study. Biological Psychiatry 47, 276-286.

Rush AJ, Marangell LB, Sackeim HA, George MS, Brannan SK, Davis SM, Howland R, Kling MA, Rittberg BR, Burke WJ, Rapaport MH, Zajecka J, Nierenberg AA, Husain MM, Ginsberg D, Cooke RG (2005a). Vagus nerve stimulation for treatment-resistant depression: a randomized, controlled acute phase trial. Biological Psychiatry 58, 347-354.

Rush AJ, Sackeim HA, Marangell LB, George MS, Brannan SK, Davis SM, Lavori P, Howland R, Kling MA, Rittberg B, Carpenter L, Ninan P, Moreno F, Schwartz T, Conway C, Burke M, Barry JJ (2005b). Effects of 12 months of vagus nerve stimulation in treatment-resistant depression: a naturalistic study. Biological Psychiatry 58, 355-363.

Rush AJ, Trivedi MH, Wisniewski SR, Nierenberg AA, Stewart JW, Warden D, Niederehe G, Thase ME, Lavori PW, Lebowitz BD, McGrath PJ, Rosenbaum JF, Sackeim HA, Kupfer DJ, Luther J, Fava M (2006). Acute and longer-term outcomes in depressed outpatients requiring one or several treatment steps: a STAR*D report. American Journal of Psychiatry 163, 1905-1917.

Sackeim HA, Rush AJ, George MS, Marangell LB, Husain MM, Nahas Z, Johnson CR, Seidman S, Giller C,
Haines S, Simpson RK, Goodman RR (2001). Vagus nerve stimulation (VNS) for treatment-resistant depression: efficacy, side effects, and predictors of outcome. Neuropsychopharmacology 25, 713-728.

Schachter SC (2002). Vagus nerve stimulation therapy summary: five years after FDA approval. Neurology 59 (Suppl. 4), S15-S20.

Schatzberg AF, Kraemer HC (2000). Use of placebo control groups in evaluating efficacy of treatment of unipolar major depression. Biological Psychiatry 47, 736-744.

Schlaepfer TE, Kosel M (2004). Novel physical treatments for major depression: vagus nerve stimulation, transcranial magnetic stimulation and magnetic seizure therapy. Current Opinion in Psychiatry 17, 15-20.

Shuchman M (2007). Approving the vagus-nerve stimulator for depression. New England Journal of Medicine 356, 1604-1607.

The Vagus Nerve Stimulation Study Group (1995). A randomized controlled trial of chronic vagus nerve stimulation for treatment of medically intractable seizures. The Vagus Nerve Stimulation Study Group. Neurology 45, 224-230.

Uthman BM (2000). Vagus nerve stimulation for seizures. Archives of Medical Research 31, 300-303.

Zobel A, Joe A, Freymann N, Clusmann H, Schramm J, Reinhardt M, Biersack HJ, Maier W, Broich K (2005). Changes in regional cerebral blood flow by therapeutic vagus nerve stimulation in depression: an exploratory approach. Psychiatry Research 139, 165-179. 